\title{
Sistemas silvopastoriles, arreglos y usos
}

\section{Silvopastoral systems, arrangements and uses}

\author{
Bueno G. Guillermo A. \\ Z. MSc. Investigador CORPOICA C.I. La Libertad. Villavicencio, Meta. Colombia. \\ gbueno@corpoica.org.co
}

Recibido11 de Julio 2012, Aprobado12 Octubre de 2012

\section{RESUMEN}

Los sistemas de producción ganadera tanto de carne como de doble propósito, con base en el ganado bovino, han sido cuestionados desde el punto de vista ambiental, en la medida que se asocian con la degradación del ecosistema causado por la deforestación para establecer pasturas. La explotación indiscriminada de los bosques es también producto del acelerado crecimiento poblacional, del mercadeo de maderas de alto valor comercial, del establecimiento de cultivos limpios y de la necesidad de conseguir leña. En consecuencia, es evidente la necesidad de desarrollar tecnologías ecológicamente sostenibles y económicamente competitivas, para prevenir el acelerado ritmo de la tumba de bosques y buscar disminuir las extensas áreas de pasturas degradadas que se han identificado en la región. Por otro lado, la región de la Orinoquia colombiana se constituye en un excelente medio para la integración del árbol en sistemas agrosilvopastoriles y silvopastoriles, donde este componente contribuye con los sistemas de producción pecuaria y agrícola. El uso de árboles multipropósito existentes en cada región, integrados con pasturas y animales en sistemas de producción, busca mejorar el nivel alimenticio y productivo de los animales, la utilización racional de los recursos y mejorar el desempeño económico y ambiental de la ganadería. Igualmente, permite romper la estacionalidad de la producción en donde la cantidad de biomasa disponible para el consumo es baja. La introducción de árboles para diferentes propósitos, responde en parte a los problemas de la deforestación y degradación del ecosistema y se proyecta como una alternativa válida para intensificar la producción y la sostenibilidad de los sistemas 
tradicionales. Así mismo, la presencia de árboles favorece a los sistemas de producción en aspectos tales como ciclaje de los nutrientes, aumento en la diversidad de especies y brinda protección física al suelo de los efectos del sol, vientos y las lluvias intensas. En los animales ofrece sombra, reduciendo el estrés por calor y radiación directa, disponibilidad de forraje en cantidad y calidad. Igualmente, reducen la evapotranspiración, la velocidad del viento y el impacto de las gotas de lluvia. Adicionalmente, la introducción de árboles se puede orientar a una reforestación o arborización de áreas degradadas y formación de corredores biológicos que a la final se traduce en una menor presión sobre los bosques primarios. De esta manera se reduce la degradación de los recursos suelo, agua y biodiversidad.

Palabras clave: Sistema silvopastoril, agrosilvopastoril, pastura.

\begin{abstract}
Livestock production systems both meat and dual purpose, based on cattle, have been questioned from the environmental point of view, as they are associated with ecosystem degradation caused by deforestation for pasture establishment. The indiscriminate exploitation of forests is also a product of rapid population growth, the marketing of high-value timber trade, the establishment of clean crops and the need to get firewood. It is therefore a clear need to develop environmentally sustainable technologies and economically competitive, to prevent the rapid pace of the tomb of forests and seek to diminish the vast areas of degraded pastures that have been identified in the region. By the other site The Colombian Orinoco region constitutes an excellent means of integrating trees in agroforestry and silvopastoral systems where this component contributes to the systems of agricultural and livestock production. Use of multipurpose trees in each region, integrated with pasture and animal production systems, aims to improve the level of food and animal production, rational use of resources and improve the economic and environmental performance of livestock. Also, lets break the seasonality of
\end{abstract}


production where the amount of biomass available for consumption is low. The introduction of trees for different purposes, partly in response to the problems of deforestation and ecosystem degradation and is projected as a valid alternative to intensify production and sustainability of traditional systems. Also, the presence of trees favors production systems in areas such as nutrient cycling, increased species diversity and provides physical protection to the floor of the effects of sun, wind and heavy rain. In animals offers shade, reducing heat stress and direct radiation, forage availability in quantity and quality. Also reduce evapotranspiration, wind speed and impact of raindrops. Additionally, the introduction of trees can be oriented to a reforestation or forestation of degraded areas and biological corridors formation that ultimately results in less pressure on primary forests. This will reduce the degradation of soil resources, water and biodiversity.

Keywords: Silvopastoral system, agroforestry, pasture.

\section{CONCEPTOS GENERALES}

Existe en la literatura una serie de definiciones de sistemas agroforestales (agroforestería), las cuales aportan elementos que permiten caracterizar el sistema. Para el tema que nos concierne se referencian algunos de importancia que integran los componentes de un sistema de producción. La agroforestería es el nombre genérico usado para designar los sistemas de uso de la tierra en los cuales las plantas leñosas perennes (árboles, arbustos y bambúes) crecen en asocio con plantas herbáceas (cultivos y pastos) y/o ganado, en un arreglo espacial, una rotación o ambos y en los cuales se dan interacciones ecológicas y económicas incluyendo los componentes arbóreos y no arbóreos del sistema (Young, 1989).

El ICRAF, (1978) define la agroforestería como un sistema sostenido del manejo de la tierra que aumenta su rendimiento total, combina la producción (incluyendo cultivos arbóreos), con especies forestales y/o animales en forma simultánea 0 secuencial sobre la misma superficie de terreno y aplica prácticas de manejo que 
son compatibles con las prácticas culturales de la población local. EI CATIE, (1998) simplifica la definición como "una forma de uso y manejo de la tierra, en la que se combina el cultivo de árboles y arbustos con cultivos alimenticios o pasto, según las prioridades del agricultor". Fassbender, (1993) indica que "los sistemas de producción agroforestales se definen como una serie de sistemas y tecnologías del uso de la tierra en los que se combinan árboles con cultivos agrícolas y/o pastos, en función del tiempo y espacio (arreglo) para incrementar u optimizar la producción en forma sostenida. Somarriba, (1998) define agroforestería como una forma de cultivo múltiple en la que se cumplen tres condiciones fundamentales: 1) Existen al menos dos especies de plantas que interactúan biológicamente, 2) al menos uno de los componentes es una leñosa perenne (árboles, arbustos y bambúes) y 3) al menos uno de los componentes es una planta manejada con fines agrícolas (incluyendo los pastos).

Los componentes de los sistemas agroforestales son:

a) Especies vegetales leñosas: son aquellas que poseen lignina como elemento de sus tejidos e incluyen; árboles, helechos arborescentes, gramíneas, cactus gigantes y arbusto como café entre otros.

b) Los no leñosos: poseen tejido vegetal poco o no lignificado, no presenta consistencia rígida, tienen porte bajo y su ciclo de vida es ligeramente inferior a un año. Este componente incluye cultivos transitorios $y$ semipermanentes, hierba y praderas.

c) El componente pecuario, incluye bovinos, ovinos, equinos, porcinos, insectos como abejas y gusanos como el de seda.

\section{CLASIFICACIÓN DE LOS SISTEMAS AGROFORESTALES EN FUNCIÓN DE LOS CULTIVOS ASOCIADOS}

Los sistemas agroforestales (SAF) existentes son complejos, diversos y flexibles., de allí la necesidad de clasificarlos en diferentes categorías, con el fin de 
evaluarlos adecuadamente y desarrollar algún plan para su mejoramiento. Según Fassbender $(1987,1993)$ y Young, (1989), los SAF se agrupan siguiendo el criterio de su base estructural, denominando cada sistema por la combinación de los tres componentes básicos que son manejados por el hombre: los leñosos perennes, plantas herbáceas (cultivos y pasturas) y el ganado, de esta manera se tiene un primer nivel de clasificación, resultando tres grupos a diferenciar de acuerdo al siguiente esquema:

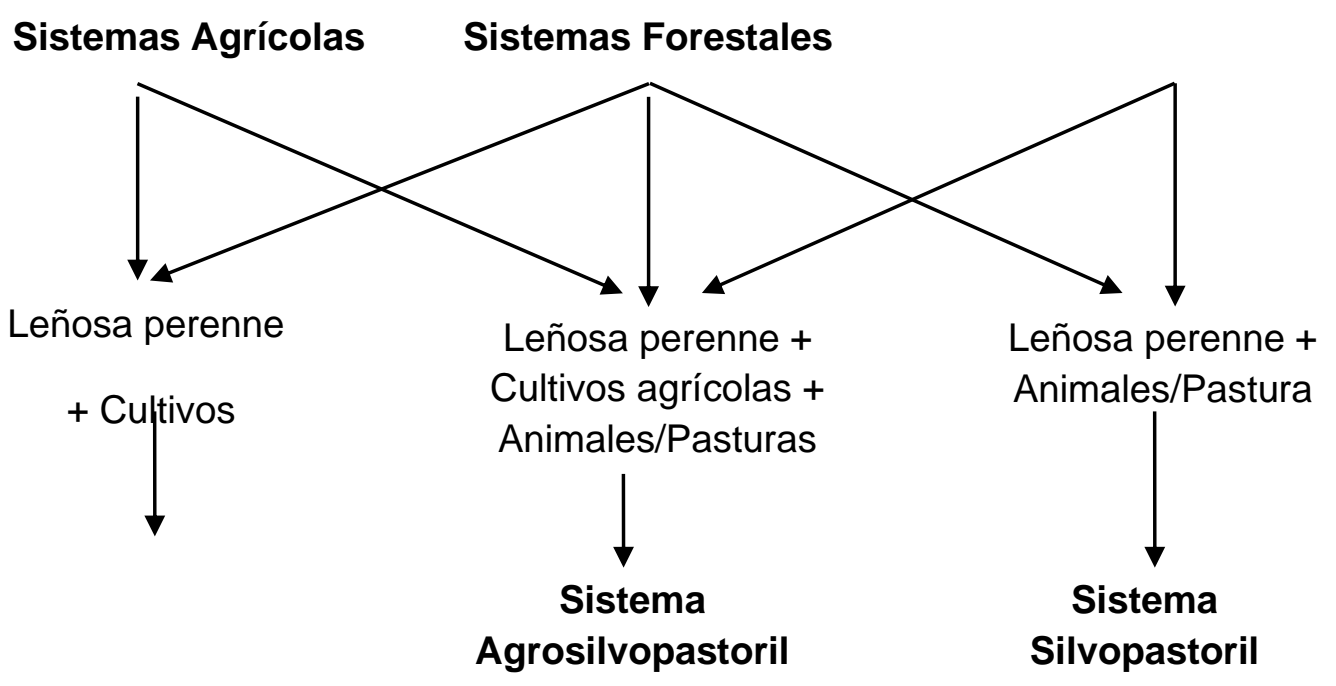

Figura 1. Clasificación de los sistemas agroforestales según su estructura

Los sistemas agroforestales o agrosilvoculturales. Son aquellos sistemas en que interactúan: leñosas perennes (árboles, frutales y arbustos) y cultivos agrícolas transitorios y semipermanentes. Corresponde a las actividades agrícolas en combinación con bosques o con árboles plantados, en los que se aprovechan los espacios que quedan entre los árboles con siembra de los cultivos agrícolas. Los sistemas agrosilvopastoriles son aquellos sistemas donde interactúan los componentes: cultivos agrícolas, árboles y ganado, con el fin de producir alimentos, forraje, madera, leña. Se aprovechan los espacios entre los árboles sembrados según arreglo forestal y se siembran los cultivos. Una vez cosechados éstos se establecen los pastos para el sistema bovino. Los sistemas silvopastoriles son sistemas que permiten la interacción entre el componente 
leñosas perennes (árboles y arbustos), no leñosos (forrajes herbáceos) y el componente animal en la misma área bajo un manejo integral. El beneficio para los sistemas tradicionales de monocultivo pastos es que ofrecen una mayor oferta de forraje y sombra para el bienestar de los animales.

Teniendo en cuenta lo anterior, la agroforestería está basada principalmente en árboles de uso múltiple, los cuales pueden hacer una contribución significativa a las funciones productivas y de servicios de los sistemas de uso de la tierra donde ellos crecen. Las principales características deseables de los árboles de uso múltiple son: 1) Existencia de uno o más productos distintos a la madera; como fuentes de forraje, leña, frutos, medicinal, estacas para cercas vivas, madera para construcción, postes, tablones. 2) Permitir el crecimiento de plantas bajo el dosel; como cultivos en callejones, sombra para el café, cacao y como soporte de otras plantas. 3) Tener efectos favorables sobre la conservación del suelo.

Además se restaura la fertilidad y estructura del suelo mediante: producción de materia orgánica; descomposición de la hojarasca; fijación de nitrógeno y fósforo; amarre del suelo; capacidad de resistir podas repetidas y buena habilidad de rebrotes; capacidad de retoñar; y capacidad de resistir el ramoneo. Aspectos económicos a través de: venta de productos; alimentación animal; flores melíferas; y abrigo para otras especies menores. Se debe tener en cuenta también los efectos perjudiciales como: efectos tóxicos en los animales; alelopatía; ramas quebradizas; y malos olores

\section{MODALIDAD DE ARREGLOS AGROFORESTALES}

Los sistemas agroforestales dada su variabilidad y flexibilidad pueden aprovecharse a diferentes escalas, según el tamaño de los predios, el nivel socioeconómico y objetivos de sus propietarios. Los árboles con cultivos pueden estar en forma dispersa, intercalados, en callejones o en líneas alternas. Pueden servir de protección utilizándolos como cercas vivas, cortinas rompe vientos, árboles en contorno y como barreras vivas. Otra forma de uso es en rodales 
compactos, bien sea como un bosque para producción de madera, un bosque energético, banco de forraje o huertos caseros. En los potreros pueden estar dispersos, en grupos (bosquetes), en línea o franjas como corredores biológicos.

Los diferentes arreglos espaciales o modalidades en sus diferentes combinaciones dan un uso adecuado de los árboles y un aprovechamiento eficiente de las áreas y del suelo. Entre los arreglos más utilizados e identificados por diferentes estudios están: plantación de árboles, arbustos y cultivos alrededor de la vivienda-huerto casero; plantación de árboles y arbustos combinados con cultivos; plantación de árboles en contorno de la finca; cercas vivas; cortinas rompevientos; árboles como sombra de cultivos permanentes; cultivos en callejones; árboles dispersos en los potreros; $y$ bosquetes en fincas (potreros).

Los anteriores arreglos espaciales son alternativas viables para las fincas y posiblemente existan otras, lo importante es conocer las características de los árboles que se ajusten a dichos modelos y del objetivo del productor. En la región de la Orinoquia (piedemonte y altillanura), se han realizado estudios a nivel de finca en los que se han identificado sistemas asociados a los cultivos (de plátano, maíz, soya, caupí, yuca y otros como pan coger) y a la producción de carne y leche. Para nuestro fin haremos énfasis en los arreglos propuestos identificados y más utilizados en los sistemas de producción bovina en la región.

Entre los arreglos agrosilvoculturales se tienen:

Árboles en cultivos agrícolas. Arreglo en que el árbol está distribuido en forma dispersa o sistemática dentro o en los bordes de las parcelas agrícolas, Figura 2. Los arreglos más frecuentes son los árboles en linderos, las cercas vivas, la cortina rompevientos y dispersos en la parcela. García (2006), encontró a nivel de fincas del piedemonte del Casanare el arreglo de árboles dispersos en bosques explotados o de regeneración natural, donde incorporaron el cultivo de plátano que cumple dos funciones: control de la enfermedad sigatoka negra en el cultivo y 
disminuir costos en insumos. Colateralmente, se logra una mejor productividad del cultivo y la obtención de otros productos como madera, leña, entre otros.

Cultivos agrícolas en callejones. El arreglo permite sembrar cultivos anuales en los callejones entre hileras de especies leñosas (árboles de rápido crecimiento) en forma paralela. Martínez y García, (2003) emplearon cono componente leñoso guandul (Cajanus cajan), Cajeto (Trichantera gigantea), matarraton (Gliricidia sepium), yopo (Piptadenia sp), Acacia mangium y Erytrina fusca, el componente agrícola lo conformaron los cultivos de yuca, maíz, caupí, arroz, soya y fríjol, materiales para uso en la alimentación humana y animal, como fuentes de proteína y energía (carbohidratos). El arreglo comprendió la siembra de árboles en surcos dobles a $0.5 \times 0.5 \mathrm{~m}$ y se dejó $8 \mathrm{~m}$ entre ellos donde se sembraron los materiales del componente agrícola. Bueno et al., (2008), en un bosquete donde el componente leñoso fue Gmelina, sembrados a $5 \mathrm{~m} \times 5 \mathrm{~m}$, se intercaló en los callejones yuca, fuente de energía en la alimentación.

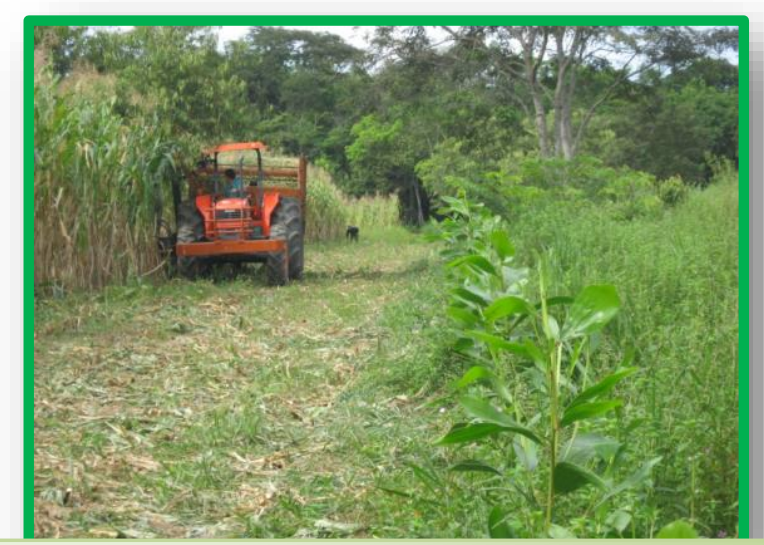

Figura 2. Árboles en línea en parcela de maíz.

Entre los arreglos silvopastoriles se han desarrollado los siguientes:

Plantación de árboles en contorno de la finca. Esta distribución espacial puede perseguir varios objetivos como la protección contra vientos, delimitación de tierras o límites de la propiedad, delimitación de potreros, función productiva (madera, alimento), protectora, confort (sombra) y embellecimiento (paisajístico). En este 
sistema se pueden combinar especies para producir madera, con especies de rápido crecimiento destinados a la producción de poste, leña, sombra (Eucalipto sp, Acacia mangium, Gmelina, Yopo, Pino caribe, frutales).

La selección de las especies vegetales dependerá del régimen de lluvias, de las condiciones del suelo y del sitio a proteger. Estas especies deben tener resistencia natural a los vientos y permanecer siempre verdes para que actúen eficientemente durante la sequía; de este arreglo se derivan otros como:

Cercas vivas. Son sistemas prácticos de cultivos de árboles, arbustos y/o palmas, que se realizan desde hace cientos de años en la zona andina y a través del tiempo han mostrado sus beneficios no solo como suministro de leña, abono verde, flores comestibles, forraje y madera para tutores de cultivos, sino que reducen considerablemente los costos de construcción y mantenimiento de las cercas divisorias de la finca y potreros (Fonseca, 1999 citados por Navas y Barragán, 2002). Consiste en sembrar árboles o arbustos en línea que sirven de soporte a las cuerdas de alambré de púas o liso para dividir potreros, delimitar la propiedad (linderos) y facilitar el uso del recurso tierra (Budowsky, 1987 citado Villanueva et al., 2008). Las cercas vivas pueden ser clasificadas en simples (una o dos especies dominantes) o multiestratos (más de dos especies leñosas de diferentes alturas), de acuerdo a la especie, estructura, alto y diámetro de copa (Murgueito et al., 2003). De acuerdo con las necesidades de protección del sistema, la especie y cantidad de sombra en potreros, la distancia entre plantas dentro del cerco vivo oscila entre 4 a $8 \mathrm{~m}$.

Estas plantas deben ser resistentes a los vientos, tolerantes a la sequía, de raíz profunda y pivotante, de rápido crecimiento, tolerante al clavado de grapas para sostener el alambrado y en lo posible que se puedan propagar por material vegetativo como estacas.

Franjas en potrero. La distribución varía de acuerdo a las especies arbóreas y del objetivo del sistema. Para el caso del silvopastoril se trazan franjas o líneas 
paralelas de 3 a 5 hileras de árboles con fines comerciales (Eucalipto, Acacia, Yopo, Melina) y se dejan los callejones con pastos entre 15 a $20 \mathrm{~m}$ para su pastoreo. Para fines ecológicos se crean corredores ambientales con franjas de 2 a 5 hileras de árboles.

Bosquete en potrero. Este arreglo consiste en hacer encierros o establecer los árboles en grupos, en un cuarto a una hectárea (estas medidas dependen del tamaño del predio o potrero que se pretende arborizar). La distancia entre árboles se diseña de acuerdo a la especie, puede variar entre 4 a $10 \mathrm{~m}$. en cuadro o tres bolillos, creando un área de fácil acceso para que los animales encuentren sombra, refugio y confort, Figura 3. Los árboles a utilizar deben permitir el paso de la luz, de rápido crecimiento, de gran tamaño y copa amplia, que no defolien en la época de verano, resistentes al ramoneo y que no sean tóxicos para el animal, los que pueden ser fuente de alimentación o de leña.

Cortinas rompevientos. El propósito es el control de la velocidad del viento para proteger cultivos, el suelo de la erosión y animales de efectos mecánicos y la desecación que producen los mismos.

Árboles dispersos en los potreros. Corresponde a un arreglo donde los árboles son sembrados a diferente distancia que pueden variar de 4 a $10 \mathrm{~m}$ o mas entre árboles, en forma desuniforme o pueden guardar estas mismas distancias en una distribución más uniforme dejando callejones donde pueden pastar los animales, Figura 4. Además, del principal beneficio de proveer sombra y ser fuente de proteína en épocas criticas (Melina, Acacia). A la vez los árboles pueden ser fuente de madera, follaje, leña, fijadores de nitrógeno atmosférico y refugio para la fauna.

Arreglo bajo plantaciones forestales. El arreglo permite utilizar las plantaciones forestales para el pastoreo de los animales, una vez se tenga la altura y buena formación o grosor del tronco para que el animal no lo dañe. 


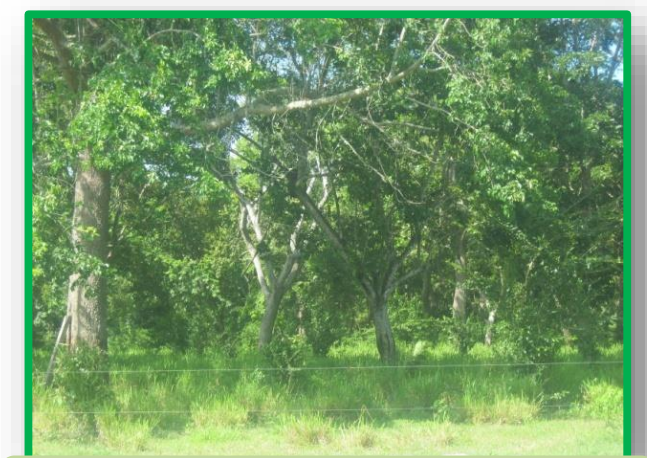

Figura 3. Bosquete en potrero

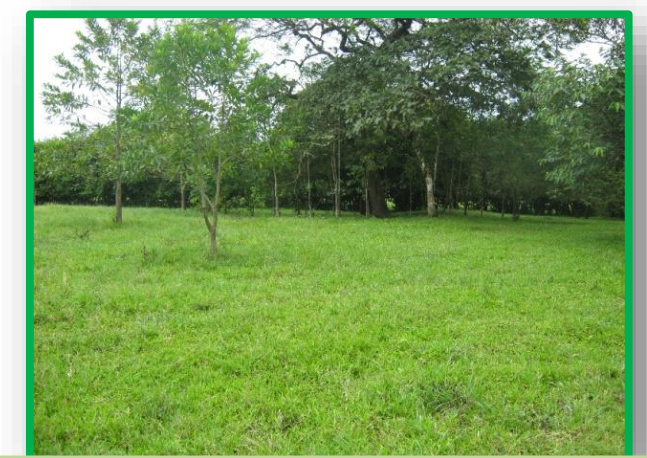

Figura 4. Árboles dispersos en el potrero

Fuente: Grupo Agroforestería

\section{ESPECIES ARBÓREAS INTEGRADAS EN SISTEMAS SILVOPASTORILES}

La Corporación Colombiana de Investigación Agropecuaria, CORPOICA, en esta última década ha venido ejecutando proyectos de investigación y transferencia de tecnología en agroforestería para la región de la Orinoquia (altillanura y piedemonte), con el fin de integrar los subsectores pecuario, agrícola y forestal como una alternativa de uso del suelo en forma sostenible y sustentable en el tiempo. El enfoque de integrar el árbol es intensificar la producción de los sistemas pecuarios y agrícolas tradicionales y a la vez diversificar plantas y animales.

En este orden, la generación de forraje y confort para el ganado sumado a la producción de madera y alimentos, incrementa la productividad de los sistemas de producción bovina. De otro lado, la integración de los componentes árbol-cultivospasto-animal, reporta beneficios en la conservación del suelo, las praderas; a nivel animal el efecto sombra reduce el estrés calórico, evitando perdida de energía en su termorregulación y por ende en el consumo de alimentos. Comparado con el monocultivo pastos, se presenta una mayor disponibilidad de alimentos en la época seca de buena calidad por sus contenidos de proteína y otros nutrientes que favorecen la fermentación ruminal y el consumo de forrajes. La estrategia busca reducir la presión sobre los bosques primarios y de galería evitando los 
actuales problemas de degradación de los recursos suelo, agua y biodiversidad que son atribuidos a la expansión de la frontera ganadera y agrícola.

En los centros de investigación, estaciones experimentales y en fincas se han establecido plantaciones y arreglos forestales usando los mismos principios de la agricultura tradicional: preparación del suelo, fertilización, siembra del arbolito, labores de mantenimiento y después de varios años (de acuerdo con la especie), cosecha de los mismos. En el proceso de investigación y validación que viene desarrollando CORPOICA en árboles y arbustos, han evaluado diversas especies en cada una de las etapas de desarrollo, desde el vivero hasta la cosecha, con el propósito de buscar alternativas tecnológicas con germoplasma seleccionado por su buen desarrollo, producción, uso, fertilización adecuada, alto grado de adaptación (plagas y enfermedades), calidad y compatibilidad con los demás componentes del sistema de producción.

\section{MANEJO DEL VIVERO}

Consideraciones técnicas a tener en cuenta para desarrollar un vivero: el número de árboles a multiplicar tiene que ver con el objetivo del productor; sistema de producción pecuario; especie de árbol a introducir; disponibilidad y semilla de buena calidad; tipo de arreglo o distribución espacial; y cantidad de sombra por potrero y animal. Lo anterior define que infraestructura y área de vivero se requiere de acuerdo al plan que debe considerar época de verano e invierno en la región, que determina la época de siembra, periodo vegetativo de la especie, cantidad de árboles a producir, método de propagación seleccionado, tipo de sustrato y envase (bolsas plásticas, tubotes o bandejas) (Figura 5). Para esta fase existen dos alternativas para la obtención del material arbóreo: adquirirlo en un vivero reconocido que garantice la calidad del material o producirlo en un vivero temporal localizado en la finca. Para el segundo caso se debe planificar el manejo del vivero y para esto existe un protocolo (Fonseca y García, 2007). Debe estar lo más cerca posible al sitio definitivo de siembra, junto a vías de fácil acceso en vehículo, con 
disponibilidad de agua durante todo el año, contar con protección perimetral contra el ingreso de animales que puedan dañar los arbolitos, una pendiente del terreno adecuada que facilite el drenaje natural de todo el vivero, adecuada circulación de aire y orientación de eras de crecimiento en dirección este - oeste, para mejor luminosidad y aprovechamiento de las horas de sol; y disponibilidad de área para posible ampliación de la producción en el vivero

Tamaño de la construcción: El tamaño, localización y distribución de la infraestructura básica del vivero depende de: sistema de propagación seleccionado; duración de los ciclos de producción de cada especie; y plan de siembras proyectado. Existen varios sistemas de producción según el sustrato utilizado: bolsas plásticas; pellets; bandejas; y conos plásticos

Sustrato o medio para la siembra de semillas arbóreas: Para este fin se recomienda la mezcla de tierra negra con arena de río o cascarilla de arroz. Estos materiales deben ser mezclados y desinfectados para el llenado de las bolsas, bandejas o conos. En este sentido se debe mezclar dos partes de tierra por una de arena, la desinfección se puede realizar mediante aplicación de agua hervida o con formol. Algunas semillas requieren de una cama o germinador con los mismos materiales que para el embolsado. Cuando las plántulas están de $5 \mathrm{~cm}$, se trasplantan a las bolsas plásticas que se colocan en el vivero y se cuidan hasta llevarlas a campo. El trasplante o siembra en sitio definitivo en el campo se realiza cuando los plantines tienen una altura entre 20 a $30 \mathrm{~cm}$.

\section{ESTABLECIMIENTO DEL ÁRBOL EN SITIO DEFINITIVO}

Inicialmente se debe seleccionar y definir la especie a integrar en el sistema productivo de acuerdo a su producto (madera, postes, leña, forraje) o servicio; para limitar áreas o linderos, sombra, cercas vivas, protección contra el viento, fines ecológicos o embellecimiento paisajístico. De otro lado, se debe considerar las experiencias que hay en la región, como de la disponibilidad de semilla 
certificada y de buena calidad, así como de tecnología para los materiales seleccionados.

El establecimiento se deberá realizar a inicio del período de lluvias. En la región de los Llanos es en el mes de Abril y se puede sembrar hasta el mes de Septiembre. Se realizará de acuerdo al arreglo espacial teniendo en cuenta un orden específico de ubicación de las especies dentro del área o lote a reforestar, se debe considerar la siembra de los árboles maderables en los sectores más retirados de los cuerpos de agua, evitando encharcamiento sobre todo para especie susceptibles a la humedad. Se recomienda que los no maderables se ubiquen en las cercanías de dichos cursos hídricos o ronda de nacimientos. Se deben cumplir con la protección perimetral dentro del establecimiento. Esto tiene que ver con dos problemas comunes en la región de un lado las quemas y de otro el daño causado por animales como el consumo de hojas y plántulas en su fase inicial de crecimiento y daño mecánico (Figura 6).

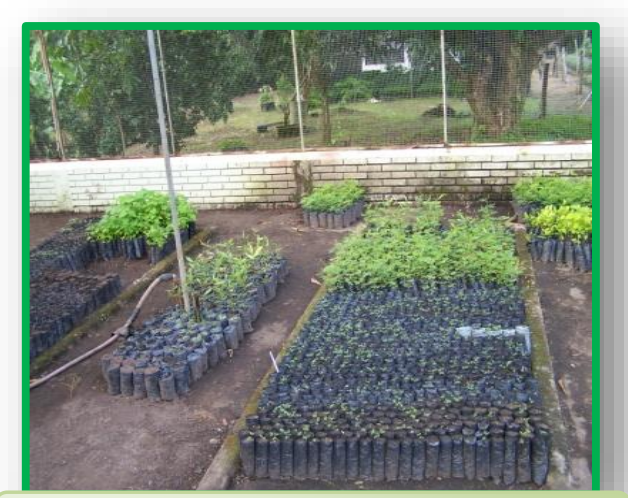

Fiqura 5. Plántulas de árboles

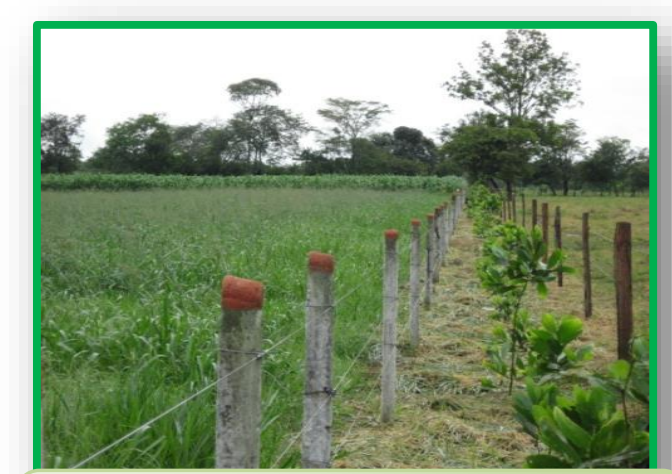

Figura 6. Cercas de protección a la línea de árboles.

Limpieza. Se debe realizar la eliminación de la vegetación herbácea (gramíneas) para evitar competencia a las plántulas por luz y nutrientes, mediante un plateo de 1 metro de radio, con azadón, machete o manualmente. Para el caso de cercas vivas de gran longitud, se recomienda hacer dos pases de rastra del ancho de trabajo del implemento; para longitudes corta o pequeñas áreas, se debe platear y remover el suelo (romper capas compactas), en un circulo con diámetro de 1 
metro. Para el caso de los bosquetes se debe controlar la vegetación mecánicamente con guadaña o rastra. Igualmente, se debe platear el sitio donde se plantará el árbol. En lotes con alta incidencia de malezas es aconsejable controlar su rebrote con herbicida.

Trazo. Los sistemas de siembra más utilizados son en línea, en cuadro, en rectángulo y en triangulo o tres bolillos. El establecimiento para cercas vivas se realiza utilizando el sistema de siembra en línea a una distancia que puede variar entre 4 a 6 metros, según la especie y la finalidad. Para el caso de franjas que pueden variar de 2 o más hileras de árboles en el que funciona bien la siembra en tresbolillo $o$ en triangulo. En los bosquetes se pueden aplicar los tres últimos sistemas de siembra y para bosque dispersos en potrero en cuadro o en rectángulo son mejores. La siembra en tresbolillo permite una mayor densidad de árboles por unidad de área que se ajusta a la distribución espacial en franjas en potrero, que consiste en sembrar 3 o más hileras y dejar callejones de 15 a 20 metros entre las franjas, que corresponde a las áreas de pastoreo mientras los árboles se desarrollan.

Las distancias de siembra varían de acuerdo a la especie, tipo de producto o función que defina el productor de acuerdo a su sistema de producción. Se calcula el número de árboles de acuerdo al sistema de siembra:

- Plantación en rectángulo se aplica la siguiente razón:

$N=10.000 / D \times d$

Donde:

$\mathrm{N}$ = Número de árboles a sembrar por hectárea

$D=$ Distancia entre filas (metro lineal)

$\mathrm{d}=$ Distancia entre árboles de una misma fila (metro lineal)

- Plantación en rectángulo:

$\mathrm{N}=10.000 / \mathrm{D} 2$

Donde:

$\mathrm{N}$ = Número de árboles a sembrar por hectárea 
$D=$ Distancia entre filas y entre surcos (metro lineal)

- Plantación en tresbolìllos:

$\mathrm{N}=(10.000 \mathrm{~m} 2 / \mathrm{D} 2) \times 1,1547$

Donde:

$\mathrm{N}$ = Número de árboles a sembrar por hectárea

$\mathrm{D}=$ Distancia entre arboles contiguos (metro lineal)

$1,1547=$ Valor constante

Ahoyado. Esta actividad depende del tipo de empaque en que se transporta la plántula. Para bolsas plásticas se deben cavar hoyos de $20 \mathrm{~cm} \times 20 \mathrm{~cm}$, con 15 a $20 \mathrm{~cm}$. de profundidad. Para el caso de los tubetes y bandejas plásticas este hoyo es de menor tamaño que para bolsas. En ambos casos, deberá hacerse un repique con barra, para romper los horizontes compactados.

Tabla 1. Distancias y densidades de siembra de árboles por hectárea, en sistemas de siembra al tresbolillo, cuadrado y rectángulo

\begin{tabular}{ccc}
\hline $\begin{array}{c}\text { Distancias empleadas según el } \\
\text { uso de las plantaciones }\end{array}$ & Tresbolillo & $\begin{array}{c}\text { En cuadro y en } \\
\text { rectángulo }\end{array}$ \\
\hline $1 \times 1$ & 11.547 & 10.000 \\
$2 \times 2$ & 2.887 & 2.500 \\
$2 \times 3$ & 1.924 & 1.666 \\
$2.5 \times 3$ & 1.540 & 1.333 \\
$3 \times 3$ & 1.283 & 1.111 \\
$3 \times 4$ & 962 & 833 \\
$4 \times 4$ & 722 & 625 \\
$5 \times 5$ & 462 & 400 \\
\hline
\end{tabular}

Fuente: Fonseca y García, (2007)

Aplicación de correctivos y primera fertilización. La actividad debe basarse en los resultados del análisis de suelo y los requerimientos nutricionales de la especie a ser plantada. Teniendo en cuenta que la característica principal de los suelos ácidos es la alta toxicidad de aluminio y deficiencia de nutrientes esenciales para el desarrollo de las plantas, como nitrógeno, fósforo, calcio y magnesio, potasio y azufre. La aplicación de fertilizantes debe cubrir y corregir estas deficiencias para lo cual se han realizado las siguientes aplicaciones con buenos resultados hasta el momento. A la siembra: cal dolomítica $150 \mathrm{~g} /$ planta; roca fosfórica $150 \mathrm{~g} /$ planta; y 
yeso agrícola $100 \mathrm{~g} /$ planta. Estos insumos se mezclan con la tierra que es sacada del hueco donde se plantará el árbol y luego se deposita en el sitio de siembra. Complementariamente, a los 15 días después de la siembra, se aplica una mezcla compuesta por: fosfato diamonico (DAP) 75 g/planta; Sulfomag 75 g/planta; y Borozinco (elementos menores) $5 \mathrm{~g} /$ planta.

Establecimiento. Una vez preparado el terreno se realizará el establecimiento o plantación de los árboles, teniendo en cuenta que: el material en bolsa debe ser embebido de agua en el momento de la siembra; se debe compactar antes de retirar la bolsa, para evitar el desmoronamiento del sustrato; la bolsa plástica se quita haciendo dos cortes longitudinales a la misma y se guarda para ser contada al final del trabajo y desechada en forma que no contamine las áreas sembradas y aledañas; las plántulas se colocan verticalmente quedando el cuello a ras del suelo, evitando la formación de depresiones que puedan afectarla por la acumulación de aguas lluvias en los mismos; y las raíces no deben quedar dobladas ni trenzadas, el tallo debe quedar vertical y la tierra se compacta con el pie, de tal forma, que la plántula quede anclada y evitar así la formación de bolsas de aire.

Todos los desechos como bolsas plásticas, estopas, costales y demás desperdicios deben ser recogidos y retirados de la zona. Se debe realizar la resiembra de todo el material que se haya perdido por diferentes causas. La mortalidad en ningún caso debe ser superior al $10 \%$.

Mantenimiento y fertilización. De acuerdo a la incidencia de plantas invasoras, se debe volver a rectificar el plateo de 1 metro de diámetro como mínimo, eliminando toda la vegetación existente dentro del plato al segundo mes de la siembra, con el objeto de eliminar competencia y preparar el terreno para la segunda aplicación de fertilizante compuesto u orgánico prehúmico o humus. El material que sea "eliminado" se puede colocar sobre el plato limpio como un mecanismo de control de malezas; seguidamente se realiza la fertilización 
incorporando al suelo el fertilizante en una cantidad de: fosfato diamonico (DAP) $150 \mathrm{~g} /$ planta; sulfomag $150 \mathrm{~g} /$ planta; y boro cinco $10 \mathrm{~g} /$ planta. En caso de fertilizante orgánico aplicar $500 \mathrm{~g} /$ planta de humus. La aplicación se realiza en forma de corona a $20 \mathrm{~cm}$ de cada plántula, y se cubre ligeramente con el sustrato existente en el predio. Considerando que se debe asegurar el desarrollo adecuado de las plantas, al tercer año se realizan labores de mantenimiento consistente en plateo de $1.50 \mathrm{~m}$ de radio, fertilización con la dosis anterior o 500 gramos de abono prehúmico, a cada individuo.

Control fitosanitario y de malezas. El control fitosanitario y de malezas deberá ser una actividad constante para evitar pérdidas del material vegetal establecido por causas de plagas y enfermedades. Para ello deben realizarse monitoreos y control como: ubicación y eliminación de hormigueros; utilización de cebos tóxicos (aserrín, melaza y un insecticida), aplicación de purines; alelopatía; control biológico y en caso extremo control químico (fungicidas e insecticidas sistémicos). Se debe mantener un control de las malezas que puedan competir por nutrientes y luz con la plantación, por ello se debe realizar el control de las mismas de forma manual, mecánico o químico.

Resiembra. Una vez realizado el establecimiento del arreglo escogido, se procederá a evaluar la mortalidad, que en ningún caso debe ser superior al 10\% del material establecido. Verificado el cultivo deberá realizar dicha resiembra, efectuando los mismos procedimientos empleados durante el establecimiento (ahoyado, encalado, plantación y fertilización).

\section{COSTOS DE ESTABLECIMIENTO DE ÁRBOLES EN ARREGLOS COMO CERCA VIVA Y BOSQUETE EN POTRERO}

Con el fin de documentar los costos en la implementación de dos arreglos silvopastoriles (bosquete en potrero y cercas vivas) en las Tablas 2 y 3 se presentan los valores para cada actividad con base en las experiencias realizadas en fincas. Para el establecimiento de especies como Acacia mangium, Yopo, 
Eucalipto, Melina, se recomienda la siembra de plantas provenientes de viveros comerciales. Se puede establecer un vivero en la finca, el manejo requerido para la producción de plantines se describió anteriormente.

Tabla 2. Costos de producción para un bosque de un cuarto de hectárea distancia de siembra 5 × $5 \mathrm{~m}$ (2010)

\begin{tabular}{|c|c|c|c|c|c|}
\hline ITEM & ACTIVIDADES MAQUINARIA Y EQUIPO & UNID & CANT & V. UNIT & V. TOTAL \\
\hline 1 & Rastra & pase & 2 & 60000 & 120.000 \\
\hline 2 & Cincel & & & & 0 \\
\hline \multirow[t]{3}{*}{3} & Encaldora & & & & 0 \\
\hline & Subtotal & & & & 120.000 \\
\hline & MANO DE OBRA & & & $\$$ & \\
\hline 1 & Alistamiento terreno & Jor & 1 & 35.000 & 35.000 \\
\hline 2 & Trazado & Und & 100 & 50 & 5.000 \\
\hline 3 & Plateo & Und & 100 & 300 & 30.000 \\
\hline 4 & Ahoyado & Und & 100 & 300 & 30.000 \\
\hline 5 & Abonado & Und & 100 & 200 & 20.000 \\
\hline 6 & Siembra & Und & 100 & 300 & 30.000 \\
\hline 7 & Resiembra & Und & 20 & 300 & 6.000 \\
\hline \multirow[t]{3}{*}{8} & Cercado & M & 200 & 800 & 160.000 \\
\hline & Subtotal & & & & 316.000 \\
\hline & INSUMOS & & 0 & & \\
\hline 1 & Plantas de $30-35 \mathrm{~cm}$ de alto & Und & 120 & 700 & 84.000 \\
\hline 2 & Calfos bulto x $50 \mathrm{Kg}$. & Bto & 1 & 15.000 & 15.000 \\
\hline 3 & Yeso agrícola x $50 \mathrm{~kg}$ & Bto & 0,5 & 18.000 & 9.000 \\
\hline 4 & Cal dolomítica x $50 \mathrm{~kg}$ & Bto & 1 & 8.000 & 8.000 \\
\hline 5 & DAP Fosfato diamónico $\times 50 \mathrm{~kg}$ & Bto & 1 & 115.000 & 115.000 \\
\hline 6 & Sulfomag x $50 \mathrm{~kg}$ & Bto & 0,75 & 25.000 & 18.750 \\
\hline 7 & Borozinco $\times 20 \mathrm{~kg}$ & Bto & 0,125 & 71.000 & 8.875 \\
\hline 8 & Alambre de púa Calibre \# 12.5 Tigre X 300 m & Buto & 2 & 180.000 & 360.000 \\
\hline 9 & Postes de madera inmunizados. (Templado) & Und & 8 & 15.000 & 120.000 \\
\hline 10 & Postes de cemento. Longitud no menor a $2.10 \mathrm{~m}$ & Und & 32 & 13.500 & 432.000 \\
\hline 11 & Grapa Tigre & $\mathrm{Kg}$ & 0,2 & 4.500 & 900 \\
\hline 12 & Alambre de amarre & $\mathrm{Kg}$ & 0,5 & 2.800 & 1.400 \\
\hline 13 & Round up & $\mathrm{L}$ & 2 & 20.500 & 41.000 \\
\hline \multirow[t]{2}{*}{14} & Blitz & $\mathrm{Kg}$ & 8 & 10.000 & 80.000 \\
\hline & Subtotal & & & & 1.293 .925 \\
\hline 1 & Muestra de suelo & Und & 1 & 75.000 & 75.000 \\
\hline 2 & Ingeniero Forestal & Global & 0,25 & 550.000 & 137.500 \\
\hline 3 & Transporte de plántulas & Und & 120 & 100 & 12.000 \\
\hline \multirow[t]{3}{*}{4} & Transporte- insumos y materiales & Global & 1 & 150.000 & 150.000 \\
\hline & Subtotal & & & & 374.500 \\
\hline & TOTAL POR BOSQUETE & & & & 2.104 .425 \\
\hline
\end{tabular}


Tabla 3. Costo (valores 2010) de $1 \mathrm{~km}$ de cerca viva

\begin{tabular}{ccc}
\hline Actividad & Valor \$ (COP) & $\%$ \\
\hline Maquinaria y equipo & 60.000 & 3 \\
Mano obra & 317.000 & 17 \\
Insumos & 286.800 & 15 \\
Cerca Eléctrica. & 830.000 & 45 \\
Otros & 350.000 & 20 \\
TOTAL & $1^{\prime} 843.800$ & 100.0 \\
\hline
\end{tabular}

\section{LOS SISTEMAS SILVOPASTORILES EN LA ACTIVIDAD GANADERA}

La creciente expansión de la actividad ganadera tiene su explicación en gran medida por que los suelos de los llanos, pobres e infértiles, han sido percibidos como recursos relativamente abundantes y que es un sector de la producción que adquiere cada vez mayor importancia en la economía de la región, donde se dedica un alto porcentaje de la superficie a este sistema de producción. Recientemente, en la región se vienen desarrollando actividades agrícolas con germoplasma más adaptado a las condiciones edafo-climáticas, bióticas y de manejo de los sistemas de producción como maíz, soya, sorgo y caña, para mejorar productividad y paralelamente tratar de conservar y mejorar el suelo en lo que se ha denominado su "capa arable". Es un componente más que juega papel importante al integrarse a los actuales sistemas agroforestales que se están implementando en la región de la Orinoquia colombiana.

La Figura 7, muestra la dinámica en el uso de las áreas de bosque en el trópico, propuesto por Giraldo, (1996). Las maderas se extraen como cultivo comercial o continuo; con los cultivos anuales se obtienen dos o tres cosechas (arroz, yuca, maíz), pero con el tiempo ambos sistemas terminan en una pastura permanente. Se debe tener presente que la ganadería bajo esta dinámica, es un punto culminante de un proceso de varios años, que se inicia con el interés comercial de explotar las maderas por su alto costo, luego vienen dos o tres años de cultivo 
limpio y sólo después que la productividad de los suelos ha disminuido, se establecen pasturas permanentes.

Se ha postulado que los sistemas silvopastoriles (SSP), en donde se combinan diversas formas de producción animal con árboles para diferentes propósitos, responden en parte a los problemas de la deforestación y degradación de los ecosistemas y a la sostenibilidad de la ganadería. Los árboles fijadores de nitrógeno aparecen como particularmente prometedores para reducir el proceso de degradación e intensificar en forma sostenible la producción de proteína de origen animal (Borel, 1987).

De otro lado, CORPOICA ha evaluado cuatro materiales arbóreos que han mostrado buen comportamiento como son: Gmelina, Acacia mangium, Eucalipto pellita y yopo, entre otros y para arbustivas se ha seleccionado Cratylia argentea, los cuales en la región del piedemonte han reportado buen comportamiento y desarrollo rápido como se resume en la Tabla 4. En evaluación realizada en CORPOICA C.I. La Libertad, se encontró similar tendencia en el crecimiento de los árboles como se resume en la Tabla 5.

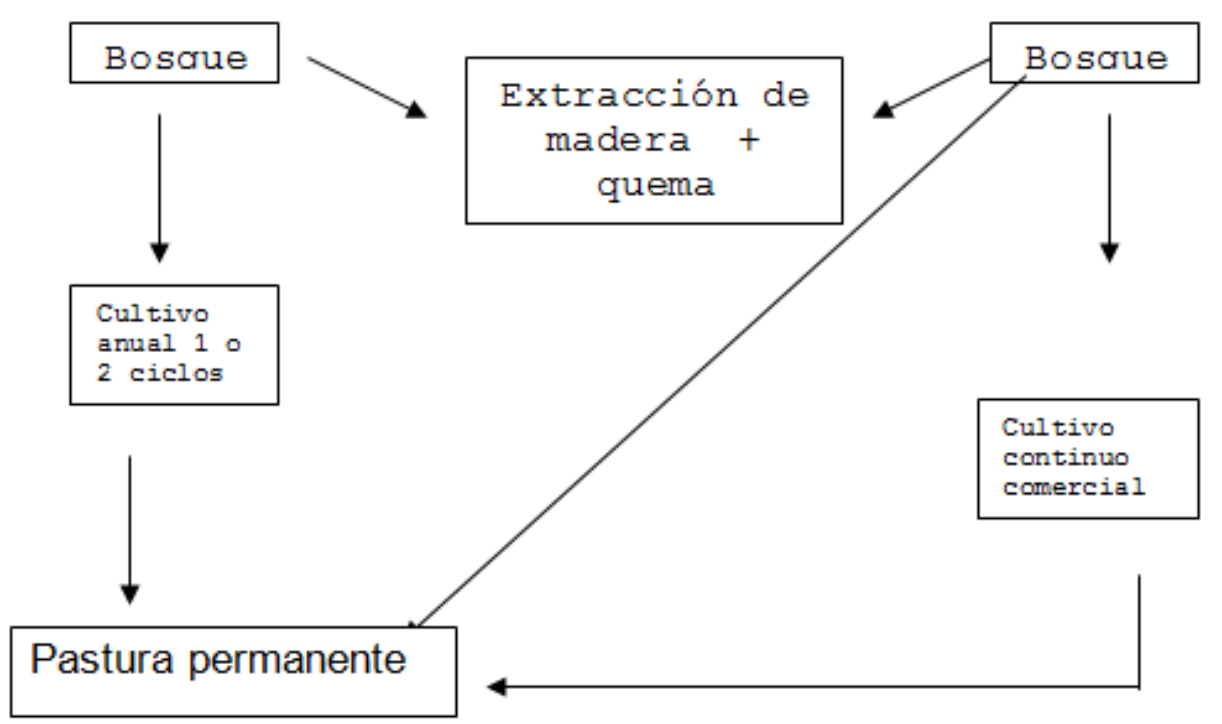

Figura 7. Esquema simplificado de los procesos dinámicos de la tala y quema de Bosques tropicales para terminar en pasturas 
De igual forma, tanto para la altillanura plana bien drenada como para la serranía, la Acacia mangium es la especie más difundida y establecida en diferentes arreglos, seguida de Eucalipto pellita. Dentro de estos arreglos se encuentran plantaciones con fines comerciales, cercas vivas, franjas y bosquetes en potrero.

Tabla 4. Altura y diámetro basal de árboles con 16 a 20 meses de sembrados en franjas, cercas vivas y bosquetes en potreros. Piedemonte llanero

\begin{tabular}{lcccc}
\hline \multicolumn{1}{c}{ Árbol } & $\begin{array}{c}\text { Número de } \\
\text { fincas* }^{*}\end{array}$ & $\begin{array}{c}\text { Promedio } \\
\text { altura }(\mathbf{m})\end{array}$ & $\begin{array}{c}\text { Rango de } \\
\text { Altura }(\mathbf{m})\end{array}$ & $\begin{array}{c}\text { DAP (cm) } \\
\text { Promedio }\end{array}$ \\
\hline Acacia mangium & 31 & 2.85 & 2.1 a 4.0 & 2.4 \\
Gmelina arbórea & 12 & 2.76 & 1.6 a 3.7 & 1.9 \\
Eucalipto pellita & 5 & 2.80 & 1.9 a 3.7 & 0.8 \\
Yopo & 13 & 2.10 & 2.0 a 2.8 & 1.3 \\
\hline * &
\end{tabular}

* 10 árboles muestreados por finca. DAP: Diámetro a la altura del pecho

Fuente: Bueno et al., (2009) (Datos sin publicar Convenio CORPOICA - ECOPETROL).

Estos materiales y sus resultados parciales indican que probablemente sea el manejo silvopastoril la combinación con más posibilidades de ser desarrollada: por tradición productora, futuro de la actividad en regiones marginales (especialmente con bovinos) y por la necesidad de reducir costos de recuperación y mantenimiento de la producción ganadera.

Tabla 5. Altura y diámetro basal de árboles después de un año de sembrados en franjas en potreros. CORPOICA C.I. La Libertad. Piedemonte llanero

\begin{tabular}{ccc}
\hline Árbol & Altura $(\mathbf{m})$ & Diámetro basal $\mathbf{( c m})$ \\
\hline Acacia mangium & 3,3 & 7,5 \\
Gmelina arbórea & 3,3 & 9,5 \\
\hline
\end{tabular}

La estructura arbórea puede ejercer su acción en diversas formas: directa sobre el animal como forrajera y como modificadora del microclima; indirecta, sobre el forraje herbáceo y arbustivo; y de apoyo a la infraestructura (postes, madera, leña, etc.). Una de las interacciones de los árboles, tiene que ver con la descomposición del material arbóreo que se deposita como detritus en el suelo, estos residuos se incorporan en la fracción orgánica del suelo y en el tiempo son absorbidos directamente por las gramíneas forrajeras. La sombra de los árboles, al atenuar la 
intensidad de luz y la temperatura foliar de las plantas, modifica también el contenido de proteínas crudas de los pastos tropicales.

De otro lado, numerosos trabajos en diferentes zonas agroecologícas del país han reportado una gran diversidad de especies con alto potencial para la alimentación animal en sistemas sivopastoriles (SSP). Simón, (1966) y Roncallo et al., (1996) plantean, en forma general, que el follaje y los frutos de las plantas nativas consumidas por los rumiantes, presentan una serie de características favorables como fuentes de alimento y permite inferir que son fuentes importantes de proteína, carbohidratos solubles, vitamina A y minerales. Sin olvidar que los principales aspectos que influyen en el contenido de nutrientes son entre otros: la especie de la planta, la fase vegetativa, parte de la planta (hojas, tallos, frutos), edad de la planta y las condiciones de su entorno (suelo, clima, etc.).

En general, el mayor potencial se encuentra en las especies leguminosas, Sin embargo, casi cualquier especie de árbol es potencialmente apta, dependiendo de las condiciones medio ambientales y socioeconómicas locales, así como de las especies a asociar, del arreglo de los componentes y de la función para la cual se incluye el valor nutritivo de los árboles varía en los diferentes componentes de la biomasa arbórea: las hojas presentan mayores concentraciones que las ramas y los tallos. La variación también se ha relacionado con la edad y con la posición en el árbol: las hojas jóvenes son más ricas que las viejas y estás además presentan porcentajes de digestibilidad bajos, debido a las concentraciones mayores de lignina y posiblemente de taninos. La Tabla 6 , resume alguno de los materiales con potencial para los sistemas silvopastoriles.

El efecto de la sombra de los árboles en los SSP, también se relaciona con el balance térmico del animal. Cuando la temperatura ambiental es inferior a la del cuerpo, el forraje consumido es metabólicamente transformado en biomasa animal, al tiempo que se genera la energía necesaria para compensar las pérdidas de calor del cuerpo. Pero cuando la temperatura del ambiente se aproxima 0 
supera la corporal, el calor metabólico generado debe eliminarse y representa un costo para el animal; la ingesta en este caso aumenta el desequilibrio térmico hasta inhibir el consumo del forraje, que conduce a la pérdida de peso corporal.

Tabla 6. Análisis nutricional de especies arbóreas

\begin{tabular}{|c|c|c|c|c|}
\hline Nombre común & Nombre científico & $\begin{array}{l}\text { P.C.. } \\
(\%)\end{array}$ & $\begin{array}{l}\text { F.D.N. }{ }^{* \star} \\
(\%)\end{array}$ & $\begin{array}{c}\text { Degradabilidad } \\
(\%)\end{array}$ \\
\hline Cratilia o Veranera & Cratylia argentea & 18.8 & 59.8 & 46.1 \\
\hline Cedro Rosado & Cedrela angustufolia & 14.4 & 55.8 & 43.4 \\
\hline Algarrobo & Hymenaca courbaril & 9.4 & 50.2 & 37.4 \\
\hline Ocobo & Tabebecia rosear & 14.4 & 48.6 & 57.9 \\
\hline Cedro Amargo & Cedrola mexicana & 17.7 & 54.8 & 31.8 \\
\hline Caño Fistol & Cassia grandis & 13.1 & 38.2 & 61.8 \\
\hline Acacia Roja & Acaccia decurrens & 16.6 & 30.4 & 54.9 \\
\hline Leucaena & Leucaena leucocephala & 17.7 & 40.8 & 50.2 \\
\hline Payande & Phithecellibium dulce & 18.5 & 48.4 & 59.0 \\
\hline Bucaro-Cachimbo & Erytrina glauca & 17.7 & 58.8 & 40.6 \\
\hline Cámbulo & Erythrina poeppigiana & 18.5 & 53.8 & 53.5 \\
\hline Lluvia de Oro & Cassia fistula & 18.1 & 52.4 & 61.5 \\
\hline Guacimo comercial & Guazuma ulmifolia & 10.7 & 39.8 & 69.2 \\
\hline Cedro Macho & Bombacopsis guinata & 11.3 & 50.4 & 45.2 \\
\hline Saman & Samanea saman & 25.1 & 35.2 & 76.0 \\
\hline Acacia & Acacia mangium & 17.0 & 34.7 & 49.9 \\
\hline Melina & Gmelina arbórea & & & \\
\hline
\end{tabular}

${ }^{*}$ PC: Proteína Cruda. ${ }^{* *}$ FDN: Fibra en detergente neutro.

Fuente: Bueno, (1998).

En los sistemas silvopastoriles, se pueden dar otras interacciones benéficas, como:

- La presencia de los árboles proporciona sombra (Figura 8) y atenúa el efecto de las temperaturas altas, que como ya se mencionó origina un ambiente más favorable para la producción y reproducción de bovinos.

- El contenido de materia orgánica y de nutrientes se incrementa en el sistema, al retornar al suelo los diferentes tipos de hojas, frutos, ramas, heces y orina. En el caso de árboles y arbustos leguminosos, habrá una contribución de nitrógeno al suelo, tanto en el fijado como en el reciclado, 
proveniente de las hojas de los árboles. Prácticas de manejo sobre la vegetación arbórea como las podas y raleos tiene efecto sobre la calidad y cantidad de los productos arbóreos (frutos, leña y madera) y el de todas las plantas del sistema.

- En árboles frutales y palmas, la limpia que hace el ganado facilita la cosecha y posterior aprovechamiento de los productos del sistema en cultivos ya establecidos.

- El pastoreo de la vegetación herbácea reduce el riesgo de incendio, especialmente en ecosistemas ubicados en zonas con época definida.

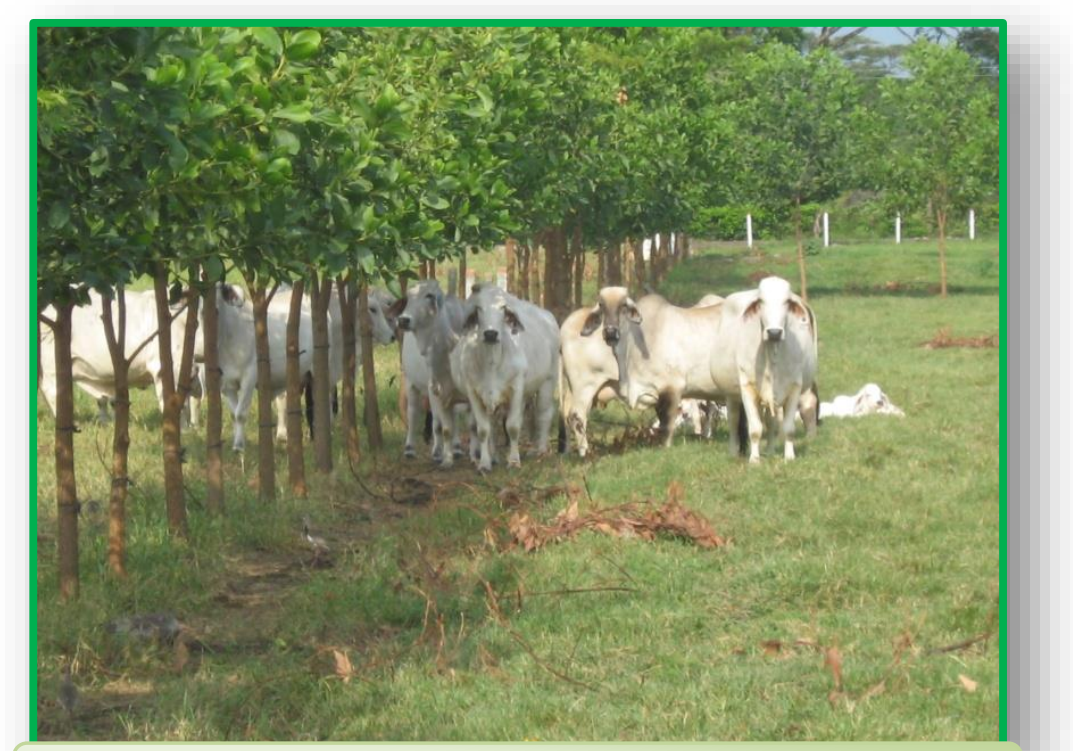

Figura 8. Árboles para la sombra de los animales

Pero, también se pueden dar interacciones negativas entre los componentes del sistema, tales como:

- La competencia por luz, debida a la sombra que los árboles ejercen sobre los estratos inferiores, afectan los rendimientos de forraje en la asociación. 
- En el caso de ecosistemas establecidos en áreas de suministros críticos de agua, la competencia por agua y nutrientes puede ser perjudicial a las herbáceas.

- El descanso y sombreo de los animales bajo los árboles, produce disminución de la cobertura herbácea y causa compactación del suelo en estos lugares.

Los actuales trabajos que integran el árbol a los sistemas de producción bovino en la región apuntan a dar confort o sombra a los animales, conservar los bosques naturales y mantener la fauna y flora. En este sentido, para estructurar la sombra se debe tener en cuenta algunas recomendaciones como:

- Orientación: se debe considerar el movimiento del sol y el ángulo de incidencia de sus rayos, tanto en verano como en invierno. Este aspecto tiene mayor importancia en explotaciones intensivas con pequeños potreros.

- Superficie: se debe disponer entre 6 y $10 \mathrm{~m}^{2}$ de sombra por animal, teniendo en cuenta la carga instantánea. Una alta concentración de animales bajo la sombra dificulta la disipación del calor corporal, destruye la pastura bajo el árbol y perjudica al propio árbol por compactación del suelo y exceso de deyecciones.

- Suelo: se deben colocar los árboles en zonas altas y/o arenosas, para evitar el encharcamiento que perjudica al animal y al árbol. En zonas de corrales se recomienda ubicar los árboles detrás de los alambrados.

- Altura: cuánto más alta es la copa, hay menor movimiento de aire y es más efectiva la neutralización de la radiación solar.

- Ventilación: no es ideal una masa arbórea continua, sino más bien pequeños grupos de árboles o individuos aislados a fin de lograr una buena 
circulación de aire. Este efecto es más importante en regiones con altas temperaturas y humedad.

- Distribución: una buena ubicación de los árboles en los potreros, constituye una de las más eficaces herramientas para mejorar la distribución del pastoreo y con ello la eficiencia de la cosecha.

\section{BIBLIOGRAFÍA}

1. Arez, M., García, F. Eucalipto. Eucalipto pellita (F Muell). Plegable divulgativo № 54. CORPOICA - CIAT. Villavicencio. Meta. 2 p. 2007.

2. Álvarez, M., García, F. Acacia. Acacia mangium (Wild). Plegable divulgativo № 54. CORPOICA - CIAT. Villavicencio. Meta. 2 p. 2007.

3. Borel, R. Sistemas silvopastoriles para la producción animal en el tropico y uso de árboles forrajeros en alimentación animal. En: Memorias VI Encuentro Nacional de Zootecnia. Cali. 24 p. 1987.

4. Bueno, G. A. Estrategias para la implementación de modelos silvopastoriles en la Altillanura Colombiana. CORPOICA - PRONATTA. Boletín Técnico № 08. Cod. 02.02.08.08.33.97. Villavicencio. Meta. Colombia. 29 p. 1997.

5. Bueno, G. A. Evaluación de especies arbóreas y alternativas forrajeras para la Altillanura Colombiana. CORPOICA - PRONATTA. Informe Técnico № 10. Villavicencio. Meta. Colombia. 63 p. 1998.

6. Centro Agronómico Tropical de Investigación y Enseñanza. CATIE. Sistemas Agroforestales. Ed: Jiménez, Vargas, A. Serie Técnica. Manual Técnico № 32. Turrialba. Costa Rica. 1998.

7. Fassbender, H. W. Modelos Edafológicos de Sistemas Agroforestales. CATIE/GTZ, Turrialba, Costa Rica. Serie de materiales de enseñanza $N^{\circ} 29$ 475. 1987.

8. Fassbender, H. W. Modelos Edafológicos de Sistemas Agroforestales. Centro Agronómico Tropical de Investigación y Enseñanza CATIE, Proyecto agroforestal CATIE/GTZ. Turrialba, Costa Rica. 1993.

9. Fonseca, I., García, F. ABeCè Forestal Herramienta para tomar decisiones. Manual Técnico No. 11. Corporación Colombiana de Investigación Agropecuaria "CORPOICA" - Gobernación del Meta. Villavicencio, Meta. Colombia. 114 p. 2007.

10. García, R. F. Sistemas agroforestales de Yopal: Diagnóstico y diseño. CORPOICA - MADR. Yopal. Casanare. 28 p. 2006.

11. Giraldo, L.A. El potencial de los sistemas silvopastoriles para la ganadería Sostenible. En: Memorias del Curso Pasturas Tropicales. Corporación Colombiana de Investigación Agropecuaria CORPOICA. Medellín. Colombia. pp. 141-172. 1996. 
12. Martínez, A., García R. F. Desarrollo de Sistemas Agroforestales para la Orinoquia Colombiana. Boletín Divulgativo № 12. CORPOICA - MADR. Villavicencio. Meta. 28 p. 2003.

13. Murgueito, E; Ibrahim, M; Ramírez, E; Zapata, A; Mejia, C; Casasola, F. Usos de la tierra en fincas ganaderas. Ed. 1. Fundación Centro para la Investigación en Sistemas Sostenibles de Producción Agropecuaria. Cali, Colombia. 97 p. 2003.

14. Navas, G., Barragán, C. Caracterización y usos potenciales de especies vegetales de un bosque de galería secundario. Boletín Técnico № 28. Corporación Colombiana de Investigación Agropecuaria "CORPOICA" y Programa Nacional de Transferencia de Tecnología "PRONATTA". Villavicencio, Meta. Colombia. 36 p. 2002.

15. Roncallo, B.; Navas, A.; Garibello, A. Potencial de los frutos de plantas nativas en la alimentación de rumiantes. En: Memorias del II Seminario Internacional sobre Sistemas Silvopastoriles; Alternativa en la ganadería. Valledupar, Neiva, Villavicencio: MADR. Diciembre de 1996. 15 p. 1996.

16. Rodríguez, R; Arias, D; Moya, R; Meza, A; Murillo, O; Arguedas, M. Manual para productores de Melina Gmelina arborea. Cartago, Costa Rica. 156 p. 2004.

17. Simón, L. Utilización de árboles leguminosos en cercas vivas y pastoreo En: Memorias del II Seminario Internacional, sistemas silvopastoriles; Alternativa en la ganadería. Villavicencio. 12 p. 1996.

18. Somarriba, E. Diagnóstico y diseño agroforestal. Agroforestería de las Américas, 5: 17-18. 1998.

19. Torres, M; Otálvaro, N; Álvarez, W; Marín, J. Identificación botánica y caracterización bromatológica de las especies arbóreas y arbustivas locales reportadas como forrajeras por productores de los municipios de Mesetas, Vista hermosa, Lejanías y Puerto Rico (Meta). CORMACARENA. Villavicencio. Meta. 150 p. 2002.

20. Villanueva, C; Ibrahim, M; Casasola, F. Valor económico y ecológico de las cercas vivas en fincas y paisajes ganaderos. Informe técnico № 372 . CATIE. Turrialba. Costa Rica. 36 p. 2008.

21. Young, A. Agroforestry for soli conservation. CAB - International ICRAF. Sciencie and Practice of Agroforestry. № 4. 276 p. 1989. 